\title{
The relationship between judgments of successiveness and judgments of order
}

\author{
LORRAINE G. ALLAN \\ McMaster University, Hamilton, Ontario, Canada
}

\begin{abstract}
The purpose of the present set of experiments was to determine the nature of the relationship between judgments of successiveness and judgments of order. The relationship was examined in terms of models which assume that the perception of successiveness is sufficient for the perception of correct temporal order. It was demonstrated that successiveness and order judgments cannot be jointly explained by perceptual latency models which assume that both judgments are based on the same internal event. This is the case regardless of the form of the perceptual latency distribution, and for either a threshold or a nonthreshold decision process. On the other hand, performance on a successiveness discrimination task, an order discrimination task, and a task requiring both a successiveness and an order judgment on each trial is consistent with the predictions of the attention-switching model.
\end{abstract}

There is a long history in experimental psychology of investigations to determine why an individual judges the order of two temporally successive events sometimes correctly, sometimes in the reverse order, and sometimes as simultaneous. However, even though numerous studies have been conducted, the source of such errors is still not established, and quantitative models which attempt to account for the errors are few (see Sternberg \& Knoll, 1973, for a summary of these models). Interest in the present paper will be focused on the attention-switching model proposed by Kristofferson (1967a, b, 1970) and elaborated by Allan and Kristofferson (1974), and on the perceptual latency models of Allan and Kristofferson (1974; Kristofferson \& Allan, 1973), Baron (1969, 1971), and Gibbon and Rutschmann (1969). While the two types of models differ in many of their basic assumptions, they share one assumption, namely that the perception of successiveness is a necessary and sufficient condition for the perception of the correct temporal order. If two successive stimulus events are perceived as such, the observer will have full information about which occurred first. Although this assumption has been explicitly stated by Allan and Kristofferson (1974) for the attention-switching model, and by Allan and Kristofferson (1974) and Barron (1969) for the perceptual latency models, it has never been directly tested.

A search of the literature yielded only two reports which provide data bearing upon the relationship

This research was supported by Grant $A 8260$ from the National Research Council of Canada. The author is indebted to the editor, Irwin Pollack, and to the referees, Jon Baron, Ronald Knoll, Ruth Rutschmann, and Saul Sternberg, for their insightful comments, and would like to express her appreciation to them for their many detailed and constructive suggestions. between judgments of successiveness and judgments of order, and the two reports differ in their conclusions. Baron (1969) presents data from one observer, whose performance can be interpreted as evidence that the source of errors in successiveness judgments is the same as that in order judgments. On the other hand, Hirsh (1959) reports that "two sounds that are separated just enough to be heard as two cannot be placed in correct order until the temporal separation is increased about tenfold" (page 767). "Whereas temporal intervals as short as a few milliseconds are sufficient to separate two brief sounds so that a listener will report that there are two (instead of only one) sounds, a longer separation time of between 15 and $20 \mathrm{msec}$ is required for the listener to report correctly which of the two sounds preceded the other " (page 759). Thus, according to Hirsh, while the perception of successiveness is necessary for the correct perception of temporal order, it is not sufficient. Hirsh's ow'n experiments were concerned with temporal order discrimination. His statements about successiveness were based on findings of others (Wallach, Newman, \& Rosenzweig, 1949), whose method, stimuli, and experimental arrangements were very different from his, and from informal observations in his own laboratory.

In the present paper, data will be presented from an experiment which was designed to investigate the relationship between successiveness judgments and order judgments. The signals were offsets of long-duration stimuli, and there were three different patterns: an $S_{1}$ pattern is one in which the offset of Stimulus $S_{A}$ precedes the offset of Stimulus $S_{B}$ by $t$ msec; an $S_{2}$ pattern is one in which the offset of $S_{B}$ precedes the offset of $S_{A}$ by $t$ msec; and an $S_{0}$ pattern is one in which the two offsets are simultaneous. During successiveness sessions, the observer was 
required to indicate whether the offsets were simultaneous or successive; during temporal order sessions, whether the offset of $S_{A}$ preceded the offset of $S_{B}$ or the offset of $S_{B}$ preceded that of $S_{A}$; and during joint sessions, he had to make a successiveness decision followed by an order decision on each trial. The successiveness sessions and the order sessions allowed the comparison of successiveness performance and order performance, each obtained separately but under identical stimulus conditions. The data from the joint sessions provide information which can be used to investigate the relationship between successiveness and order judgments on a particular trial.

\section{METHOD}

Three paid observers participated in the experiment. Their task was to judge the temporal relationship between the offset of a light $\left(S_{A}\right)$ and the offset of a tone $\left(S_{B}\right)$. Each observer was seated in a dimly illuminated, IAC sound-attenuated auditory chamber, approximately $66 \mathrm{~cm}$ from the visual display. A glow modulator bulb (Sylvania R1131C), driven by an Iconix power supply (Model 6195-4), was used to generate a 50-fL light signal. The bulb was enclosed in a metal box with an aperture, $4 \mathrm{~mm}$ in diam, subtending a visual angle of approximately $21 \mathrm{~min}$. The auditory signal was a $810-\mathrm{Hz}, 72-\mathrm{dB}$ pure tone. generated by a Wavetek function generator and presented to the observer through earphones. The presentation and timing of the signals, and the recording of the responses were under the control of a PDP-8/E computer.

On each trial, one light-tone pair was presented. The onsets of the light and the tone were always physically simultaneous. On one third of the trials, the offsets were simultaneous (an $S_{0}$ pattern), on one third of the trials, the light terminated before the tone (an $S_{1}$ pattern), and on one third of the trials, the tone terminated before the light (an $S_{2}$ pattern). When an $S_{0}$ pattern was presented, the external or physical duration of both the light and the tone was $2,000 \mathrm{msec}$. When an $S_{1}$ pattern was presented, the duration of the light was always $2,000 \mathrm{msec}$ and the duration of the tone was 2,025, $2,050,2.075$, or $2.100 \mathrm{msec}$. That is, $t$ (the temporal interval between the offset of the light and the offset of the tone) was 25,50 . 75 , or $100 \mathrm{msec}$. The four values of $t$ were equally likely. When an $\mathrm{S}_{2}$ pattern was presented, the duration of the tone was $2,000 \mathrm{msec}$ and the duration of the light was 2,025, 2,050,2,075, and $2,100 \mathrm{msec}$. Again, the four values of $t,-25,-50,-75$, and $-100 \mathrm{msec}$, were equally likely. A negative value of $t$ indicates that tone offset occurred prior to light offset.

An experimental session consisted of three blocks of 96 trials each, with a 1 -min rest between blocks. During each block, $32 \mathrm{~S}_{0}$ patterns, $32 S_{1}$ patterns, and $32 S_{2}$ patterns were presented in a random order. Of the $32 S_{1}$ patterns. there were 8 at each of the four levels of $t$; similarly for the $S_{2}$ patterns.

The response period began $1.5 \mathrm{sec}$ after the termination of the stimulus pattern, and was marked by the on period of a visual cue. During successiveness sessions, the observer was given $2 \mathrm{sec}$ to indicate whether the offsets were successive $\left(R_{S}\right)$ or simultaneous $\left(R_{0}\right)$. During temporal order sessions, he was given $2 \mathrm{sec}$ to ind icate whether the light terminated before the tone $\left(R_{1}\right)$ or the tone before the light $\left(R_{2}\right)$. During joint sessions, he was given $1.5 \mathrm{sec}$ to respond $R_{S}$ or $R_{0}$ and then $1.5 \mathrm{sec}$ to respond $R_{1}$ or $R_{2}$. The interval between the end of the response period and the onset of the next stimulus pattern was $1.5 \mathrm{sec}$. At no time during the experiment was the observer given any information regarding his performance, other than that related to consistency and stability.
Two observers, T.M. and A.J., were exposed to the joint task (four sessions), then the successiveness task (three sessions), and then the order task (six sessions). For B.P., the sequence was order (five sessions), successiveness (four sessions), and joint (five sessions). The observers received varying amounts of practice before each type of session.

\section{RESULTS AND DISCUSSION}

For each observer, the summary statistics of interest are presented in Table 1 for each session type. For the successiveness sessions, the probability of a successive response, $P\left(R_{S}\right)$, is shown; for the order sessions, the probability of an $R_{1}$ response, $P\left(R_{1}\right)$; and for the joint sessions, the probability of each of the four possible response combinations, $P\left(R S \cap R_{1}\right)$, $P\left(R_{0} \cap R_{1}\right)$, $P\left(R S \cap R_{2}\right)$, and $P\left(R_{0} \cap R_{2}\right)$, the marginal probability of a successive response, $P\left(R_{S}\right)$, the marginal probability of an $R_{1}$ response, $P\left(R_{1}\right)$. and the probability of an $R_{1}$ response conditionalized on a successive response, $P\left(R_{1} \mid R_{S}\right)$.

Examination of the nonzero $t$ values shows that during the joint sessions the probability of a correct order response conditionalized on a correct successive response, $P\left(R_{j} \mid R S \cap S_{j}\right)$ for $j$ equal to 1 or 2 , is larger than the unconditional probability of a correct order response, $P\left(R_{j} \mid S_{j}\right)$, in 19 of the 24 possibilities (eight $t$ values and three observers). The null hypotheses of independence between successive and order judgments can be rejected $\left(\chi^{2}=8.17\right.$, $\mathrm{p}<.005)$. The data indicate that order judgments in the joint task are related to successiveness judgments.

In order to determine the nature of the dependency of order judgments on successiveness judgments, assumptions about the source of errors in these types of judgments, and about the observer's response biases and preferences have to be made. The approach taken in this paper is to compare the data to the predictions of models which assume that the perception of successiveness is sufficient for the perception of correct temporal order. Two such models were evaluated, the perceptual latency model and the attention-switching model.

\section{Perceptual Latency Models}

The common feature of the perceptual latency models is that the lag between the occurrence of a stimulus event and its perception is assumed to be variable. It is this variability in perceptual latency that results in errors in the judgments of successiveness and order. Stimulus $S_{A}$ is presented prior to Stimulus $S_{B}$. However, depending upon the perceptual lag times, sometimes $S_{A}$ is perceived prior to $S_{B}$, sometimes after $S_{B}$, and sometimes simultaneously with $\mathrm{S}_{B}$. The various perceptual latency models differ in their assumptions about the 
Table 1

Summary Data for Each Observer for Each Session Type

\begin{tabular}{|c|c|c|c|c|c|c|c|c|c|c|}
\hline \multirow[b]{2}{*}{ Observer } & \multirow[b]{2}{*}{$\mathrm{t}$} & \multirow{2}{*}{$\begin{array}{c}\text { Successiveness } \\
\text { Sessions } \\
\text { P( }\left(\mathbf{R}_{\mathbf{S}}\right) \\
\end{array}$} & \multirow{2}{*}{$\begin{array}{c}\text { Order } \\
\text { Sessions } \\
P\left(R_{1}\right) \\
\end{array}$} & \multicolumn{7}{|c|}{ Joint Sessions } \\
\hline & & & & $\begin{array}{c}P\left(R_{S} \cap\right. \\
\left.R_{1}\right) \\
\end{array}$ & $\begin{array}{c}P\left(R_{O} \cap\right. \\
\left.R_{1}\right)\end{array}$ & $\begin{array}{c}\mathbf{P}\left(\mathbf{R}_{\mathbf{S}} \cap\right. \\
\left.\mathbf{R}_{2}\right)\end{array}$ & $\begin{array}{c}P\left(R_{O} \cap\right. \\
\left.R_{2}\right)\end{array}$ & $\mathbf{P}\left(\mathbf{R}_{\mathbf{S}}\right)$ & $\mathbf{P}\left(\mathbf{R}_{\mathbf{1}}\right)$ & $\mathbf{P}\left(\mathbf{R}_{\mathbf{1}} \mid \mathbf{R}_{\mathrm{S}}\right)$ \\
\hline \multirow{9}{*}{ T.M. } & -100 & .82 & .08 & .00 & .04 & .91 & .04 & .92 & .04 & .00 \\
\hline & -75 & .74 & .17 & .07 & .06 & .79 & .07 & .86 & .14 & .08 \\
\hline & -50 & .47 & .23 & .13 & .17 & .58 & .09 & .73 & .32 & .18 \\
\hline & -25 & .22 & .30 & .13 & .28 & .36 & .21 & .50 & .42 & .26 \\
\hline & 0 & .07 & .57 & .08 & .38 & .14 & .40 & .22 & .46 & \\
\hline & 25 & .02 & .62 & .13 & .47 & .08 & .30 & .22 & .61 & .59 \\
\hline & 50 & .19 & .69 & .31 & .41 & .06 & .20 & .38 & .73 & .82 \\
\hline & 75 & .57 & .85 & .66 & .17 & .09 & .06 & .76 & .84 & .87 \\
\hline & 100 & .87 & .90 & .89 & .01 & .05 & .04 & .95 & .91 & .94 \\
\hline \multirow{9}{*}{ A.J. } & -100 & .96 & .04 & .06 & .01 & .91 & .01 & .98 & .07 & .06 \\
\hline & -75 & .62 & .13 & .15 & .06 & .76 & .02 & .92 & .22 & .16 \\
\hline & -50 & .50 & .25 & .11 & .12 & .62 & .13 & .74 & .24 & .15 \\
\hline & -25 & .36 & .35 & .21 & .21 & .37 & .18 & .59 & .44 & .36 \\
\hline & 0 & .16 & .48 & .21 & .29 & .24 & .26 & .45 & .50 & \\
\hline & 25 & .28 & .61 & .26 & .31 & .21 & .20 & .48 & .57 & .54 \\
\hline & 50 & .51 & .78 & .39 & .30 & .16 & .13 & .56 & .70 & .70 \\
\hline & 75 & .69 & .78 & .67 & .10 & .15 & .06 & .83 & .78 & .81 \\
\hline & 100 & .93 & .86 & .83 & .01 & .13 & .02 & .97 & .84 & .86 \\
\hline \multirow{9}{*}{ B.P. } & -100 & .95 & .02 & .10 & .04 & .84 & .02 & .94 & .14 & .11 \\
\hline & -75 & .85 & .05 & .17 & .12 & .66 & .04 & .83 & .30 & .20 \\
\hline & -50 & .72 & .18 & .16 & .16 & .60 & .08 & .76 & .32 & .21 \\
\hline & -25 & .53 & .45 & .17 & .25 & .38 & .20 & .54 & .42 & .31 \\
\hline & 0 & .30 & .65 & .21 & .36 & .25 & .18 & .46 & .57 & \\
\hline & 25 & .31 & .64 & .32 & .26 & .24 & .18 & .56 & .58 & .57 \\
\hline & 50 & .38 & .78 & .32 & .27 & .21 & .20 & .54 & .59 & .59 \\
\hline & 75 & .52 & .75 & .58 & .23 & .10 & .09 & .68 & .81 & .85 \\
\hline & 100 & .75 & .81 & .73 & .06 & .12 & .09 & .85 & .79 & .86 \\
\hline
\end{tabular}

form of the perceptual latency distribution and about the nature of the decision process.

The function $f(I)$ is the convolution of two perceptual offset latency distributions and represents the distribution of the temporal difference between the perceived offsets of the two stimuli. On each trial, the value of $I$ is compared to a criterion value, and the decision is based on the outcome of this comparison process. If it is assumed that the same internal observation (a value of I) is used for both successiveness and order judgments on a given trial, then, for the joint task, a nonthreshold decision process would represent the observer as adopting three criteria, $\mathrm{C}_{2}<\mathrm{C}<\mathrm{C}_{1}$. If the perceptual difference between the offsets of $S_{A}$ and $S_{B}$ is a large positive value, the observer decides that an $S_{1}$ pattern was presented and he responds $R_{S}$ followed by $R_{1}$; if the difference is a large negative value, he decides that an $S_{2}$ pattern was presented and he responds $R_{S}$ followed by $R_{2}$. The remaining intermediate values are categorized as simultaneous. Since the observer is required to make an order response even when he has labeled a pattern as simultaneous, he divides the simultaneous responses so that the more positive values are labeled $R_{1}$ and the more negative as $R_{2}$.

As $t$ is varied in the joint task, three probability functions, $P\left(R_{S} \cap R_{1}\right), P\left(R_{1}\right)$, and $1-P\left(R S \cap R_{2}\right)$, are generated. Regardless of the form of the difference distribution, a perceptual latency model incorporating a nonthreshold decision process with three criteria requires the three probability functions to be distinguished by horizontal translation only. In Figure 1, a set of three functions generated from a normal $\mathrm{f}(\mathrm{I})$ distribution are illustrated. The $P\left(R_{S} \cap R_{1}\right)$ function is displaced $25 \mathrm{msec}$ to the right of the $P\left(R_{1}\right)$ function, and the $1-P\left(R_{S} \cap R_{2}\right)$ function $50 \mathrm{msec}$ to the left.

In Figure 2, the obtained values of $P\left(R_{S} \cap R_{1}\right)$, $P\left(R_{1}\right)$, and $1-P\left(R_{S} \cap R_{2}\right)$ are plotted as a function of $t$ for each observer. The three probability functions are not simply horizontally displaced. This is more readily demonstrated by assuming that $f(I)$ is normally distributed and transforming the probability values to standard scores, $\mathrm{Z}\left(\mathrm{RS}_{\mathrm{S}} \cap \mathrm{R}_{1}\right), \mathrm{Z}\left(\mathrm{R}_{1}\right)$, and $\mathrm{Z}\left(\mathrm{R}_{2} \cap \mathrm{R}_{\mathrm{S}}\right)$. The three transformed functions should be linear and parallel. In Figure 3, the standard scores, averaged over the three observers, are shown as a function of $t$. The functions deviate from linearity and are not parallel.

It is clear that the data are not consistent with a nonthreshold perceptual latency model, which asserts that the same internal observation on the same unidimensional scale is used for both successiveness and order judgments on a given trial. Let us now 


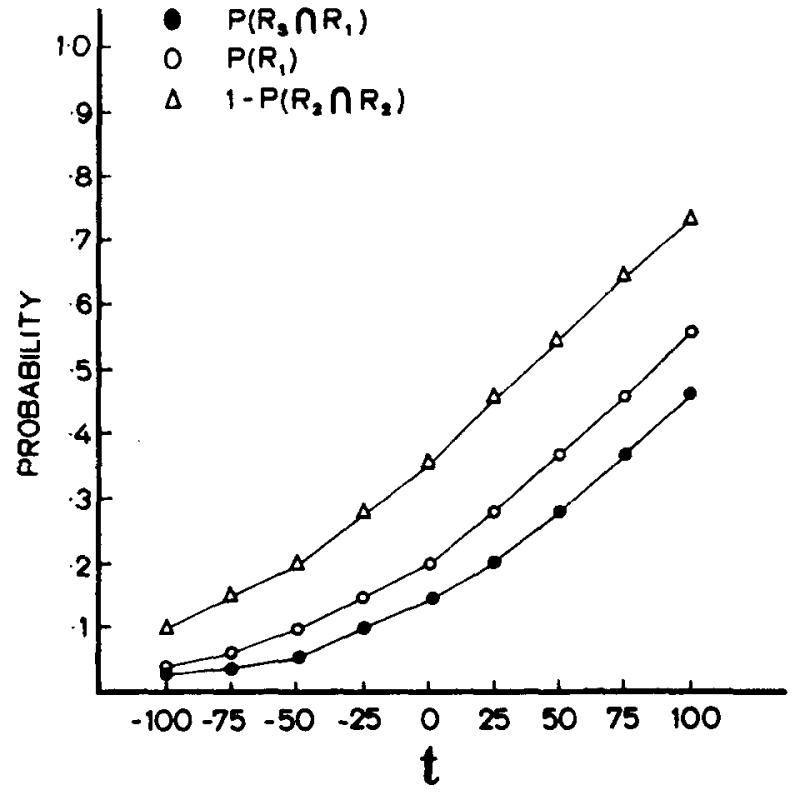

Figure 1. Probability functions generated by a nonthreshold, perceptual latency model, assuming f(I) to be normally distributed.
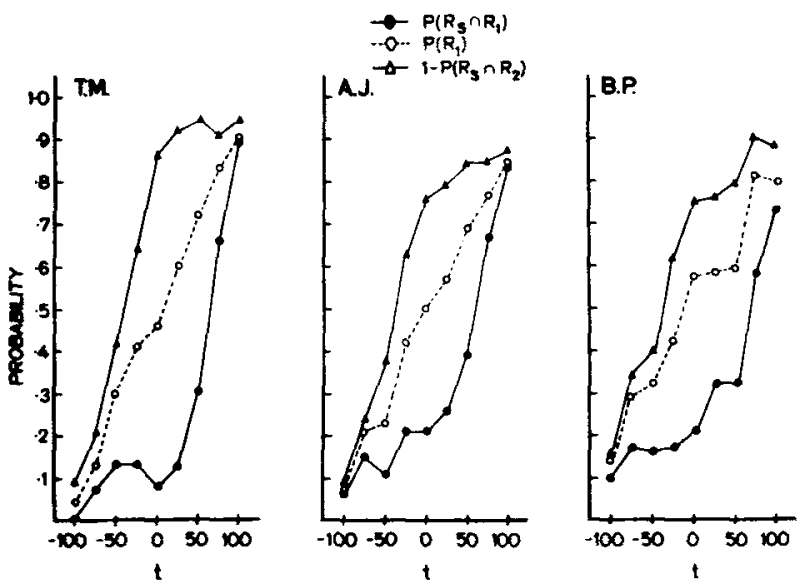

Figure 2. $P\left(R_{S} \cap R_{1}\right), P\left(R_{1}\right)$, and 1 - $P\left(R_{S} \cap R_{2}\right)$ as a function of $t$ for each observer.

consider a threshold decision process with two criteria, $\mathrm{C}_{2}<\mathrm{C}_{1}$ (Sternberg \& Knoll's Model 3). If $\mathrm{C}_{2}<\mathrm{I}<\mathrm{C}_{1}$, no order information is available and the observer always responds $R_{0}$. Since he is required to make an order response on such trials, he makes an $R_{1}$ response with probability $\beta$. If $I \geqslant C_{1}$, the observer responds $R S \cap R_{1}$; if $I \leqslant C_{2}$, he responds $R S \cap R_{2}$. The threshold decision process requires two of the probability functions, $P\left(R_{S} \cap R_{1}\right)$ and 1 $P\left(R S \cap R_{2}\right)$, to be horizontally displaced. Again, Figures 2 and 3 show this not to be the case.

In conclusion, the data from the joint sessions are not consistent with perceptual latency models if it is assumed that the same internal observation on the same unidimensional scale is used for both successiveness and order judgments on a given trial.
This is the case for nonthreshold and threshold decision processes.

\section{The Attention-Switching Model}

The attention-switching model postulates that the processing of successiveness information is under the control of an internal timing mechanism which generates a succession of equally spaced points in time. These points occur at the rate of one every $\mathrm{q}$ msec, and their occurrence is independent of the presentation of an external stimulus. It is assumed that there are independent input channels, that only one channel can be attended to at a time, and that attention can be switched from one channel to another only at an internally generated time point. For the perception of successiveness, at least one time point must fall in the interval between the two stimuli. Thus, the source of errors in judgments of successiveness is the waiting time before attention can switch from one channel to the other. The waiting time has a uniform distribution over a range from 0 to $q$ msec.

If $x$ represents the amount by which the conduction time of $S_{B}$ is faster than that of $S_{A}$, then the probability that a time point will fall between two offsets is $\alpha$, where

$\alpha= \begin{cases}\frac{t-x-n q}{q} & \text { for } x+n q \leqslant t \leqslant x+(n+1) q \\ \frac{-(t-x)-n q}{q} & \text { for } x-(n+1) q \leqslant t \leqslant x-n q\end{cases}$

and $\mathbf{n}$ is a nonnegative integer.

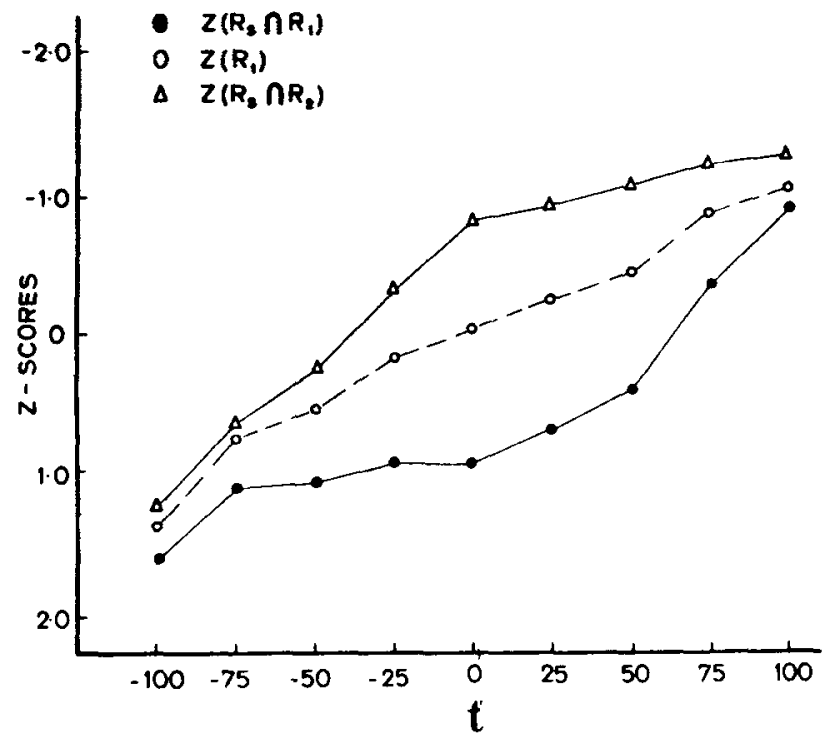

Figure 3. $Z\left(R_{S} \cap \mathbf{R}_{1}\right), Z\left(R_{1}\right)$, and $Z\left(R_{S} \cap R_{2}\right)$ as a function of $t$. 


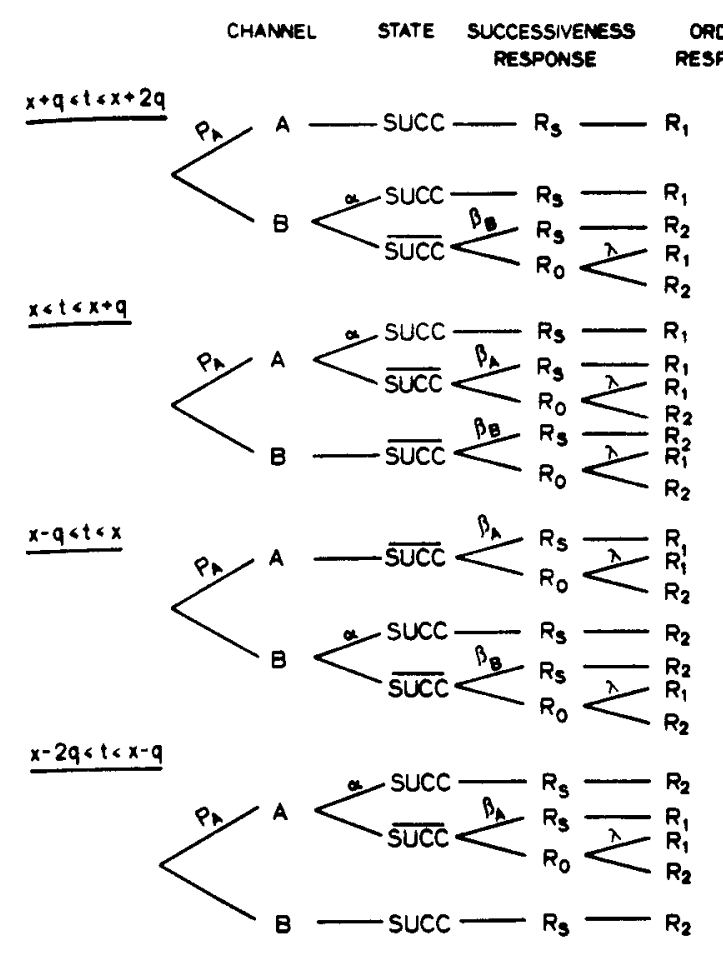

Figure 4. Schematic of the attention-switching model.

In Figure 4, the attention-switching model, for $\mathrm{x}-2 \mathrm{q} \leqslant \mathrm{t} \leqslant \mathrm{x}+2 \mathrm{q}$, is presented schematically. The parameter, $P_{i}$, for $i$ equal to $A$ or $B$, represents the observer's preference for Channel A and Channel B, respectively. It is assumed that the observer is always attending to one of the two relevant channels, $\Sigma \mathrm{P}_{\mathrm{i}}=1.0$, and that he will switch to the nonattended channel at the next opportunity. If the observer is attending to the channel of the first offset when it occurs, one time point is required for him to be able to switch to the channel of the second offset before it occurs; if he is attending to the inappropriate channel at the time of the first offset, two time points are required in order to switch to the channel of the second offset before it occurs. If the observer is able to switch to the appropriate channel before the occurrence of the second offset, he will know the two offsets were successive and he will make a correct response, $\mathrm{RS}$, with probability 1.0 ; if he is not able to, he will not have any information about the temporal relationship between the two offsets, and he will make an $\mathrm{RS}$ response with probability $\beta_{\mathrm{i}}$, for $\mathrm{i}$ equal to $A$ or $B$. The value of the bias parameter, $\beta_{i}$, depends upon the channel preference. For example, consider $x \leqslant t \leqslant$ $x+q$. The offset of $S_{A}$ precedes the offset of $S_{B}$. If the observer is attending to Channel $A$ when $S_{A}$ occurs, then with probability $\alpha$ he will be able to switch to Channel $B$ before $S_{B}$ occurs, he will know $S_{A}$ occurred prior to $S_{B}$, and he will make an $R_{S}$ response; with probability $(1-\alpha) \mathrm{S}_{\mathrm{B}}$ will have occurred prior to the switch, he will not have any information about the temporal relationship between the two offsets, and he will make an RS response with probability $\beta \mathrm{A}$. If the observer is attending to Channel $B$ when $S_{A}$ occurs, he will never have any information about the temporal relationship between the two offsets, and he will make an RS response with probability $\beta_{\mathrm{B}}$.

The relationship between $P(R S)$ and $t$ is shown in Table 2. For $x-2 q \leqslant t \leqslant x+2 q$, the successiveness function consists of four linear segments, each segment spanning $q$ msec. In order to reduce the number of parameters, $\beta_{A}$ was set equal to $\beta_{B}\left(\beta_{A}=\right.$ $\left.\beta_{\mathbf{B}}=\beta\right)$. For each observer, values of the four parameters, $q, x, P_{A}$, and $\beta$, which minimized the sum of the squared deviations between the obtained values of $P(R S)$ and those predicted by the model were determined. In previous studies of successiveness using similar light-tone pairs, $\mathrm{x}$ was always positive and $\mathrm{q}$ was estimated to be around $50 \mathrm{msec}$. Accordingly, $x$ was varied from 0 in a positive direction in integer increments and $\mathrm{q}$ was varied in both directions, starting at $50 \mathrm{msec}$ in integer increments (decrements). The parameters $P_{A}$ and $\beta$ were varied between 0 and 1 in .01 increments. The parameter values for the successiveness sessions for each observer, along with the proportion of the total variance in $\mathrm{P}\left(\mathrm{RS}_{\mathrm{S}}\right)$ accounted for by the model, are presented in Table 3. For observer T.M., the estimated value of $\beta$ is zero, implying that when he had no successiveness information he always made an $\mathrm{R}_{0}$ response. Since this observer had such a strong bias for simultaneous responses, it is conceivable that he made an $R_{0}$ response with probability $\beta^{\prime}$ even when he perceived the stimuli as successive. New parameter values were determined for this observer, assuming that

$$
\mathrm{P}(\mathrm{RS} \mid \text { succ })=\beta^{\prime} \leqslant 1.00 \text {. }
$$

These values are also available in Table 3 .

In Figure 5a, $\mathrm{P}\left(\mathrm{R}_{\mathrm{S}}\right)$ from the successiveness sessions is plotted as a function of $t$. The lines represent the functions based on the parameter values in Table 3. It is clear that the model provides a good account of the data. All other published tests of the attention-switching model by Kristofferson and by Allan have been with successiveness data from a

Table 2

The Attention-Switching Model Successiveness Function

\begin{tabular}{ll}
\hline \multicolumn{1}{c}{$t$} & \multicolumn{1}{c}{$\mathbf{P}\left(\mathbf{R}_{\mathbf{S}}\right)$} \\
\hline $\mathrm{x}+\mathrm{q} \leqslant \mathrm{t} \leqslant \mathrm{x}+2 \mathrm{q}$ & $\alpha\left(1-\mathrm{P}_{\mathbf{A}}\right)\left(1-\beta_{\mathbf{B}}\right)+\beta_{\mathbf{B}}\left(1-\mathbf{P}_{\mathbf{A}}\right)+\mathbf{P}_{\mathbf{A}}$ \\
$\mathrm{x} \leqslant \mathrm{t} \leqslant \mathrm{x}+\mathrm{q}$ & $\alpha \mathbf{P}_{\mathbf{A}}\left(1-\beta_{\mathbf{A}}\right)+\mathbf{P}_{\mathbf{A}}\left(\beta_{\mathbf{A}}-\beta_{\mathbf{B}}\right)+\beta_{\mathbf{B}}$ \\
$\mathrm{x}-\mathrm{q} \leqslant \mathrm{t} \leqslant \mathrm{x}$ & $\alpha\left(1-\mathbf{P}_{\mathbf{A}}\right)\left(1-\beta_{\mathbf{B}}\right)+\mathbf{P}_{\mathbf{A}}\left(\beta_{\mathbf{A}}-\beta_{\mathbf{B}}\right)+\beta_{\mathbf{B}}$ \\
$\mathrm{x}-2 \mathrm{q} \leqslant \mathrm{t} \leqslant \mathrm{x}-\mathrm{q}$ & $\alpha \mathbf{P}_{\mathbf{A}}\left(1-\beta_{\mathbf{A}}\right)+\beta_{\mathbf{A}} \mathbf{P}_{\mathbf{A}}+\left(1-\mathbf{P}_{\mathbf{A}}\right)$ \\
\hline
\end{tabular}


Table 3

Estimates of Parameters of Attention-Switching Model

\begin{tabular}{clccccccccc}
\hline Observer & Session Type & $\mathrm{q}$ & $\mathrm{x}$ & $\mathrm{P}_{\mathbf{A}}$ & $\beta_{\mathbf{A}}$ & $\beta_{\mathbf{B}}$ & $\beta^{\prime}$ & $\lambda$ & $\theta$ & Proportion \\
\hline & Successive & 60 & 0 & .36 & .00 & .00 & & & & .940 \\
T.M. & Successive & 51 & 0 & .31 & & & .83 & & & .955 \\
& Joint & 54 & 0 & .35 & .28 & .15 & & .57 & .53 & .977 \\
& Order & & & .39 & & & & & .975 \\
A.J. & Successive & 56 & 4 & .56 & .12 & .12 & & .5 & & .979 \\
& Joint & 56 & 0 & .39 & .49 & .33 & & .53 & .49 & .979 \\
& Order & & & .47 & & & & & & .997 \\
B.P. & Successive & 62 & 5 & .27 & .25 & .25 & & .60 & & .974 \\
& Joint & 62 & 0 & .39 & .50 & .36 & & .60 & .68 & .973 \\
\hline
\end{tabular}

forced-choice task employing positive values of $t$ only. The present data indicate that the model can successfully account for successiveness data obtained in a single stimulus task using both positive and negative values of $t$.

While the assumption that the perception of successiveness is a necessary and sufficient condition for the perception of correct temporal order has been explicitly stated by Allan and Kristofferson (1974), the model has never been applied to order data. The schematic in Figure 4 shows a possible extension of the model to represent the observer's behavior when he is required to make an order response following a successive/simultaneous response. On trials when the observer has order information, he will make a correct order response; on trials when no order information is available and an $R_{0}$ response was made, an $R_{1}$ response is made with probability $\lambda$; on trials when no order information is available but an RS response was made, the order response is determined by the channel preference on that trial.

The relationship between three of the joint probabilities and $t$ is shown in Table 4. Consider first the two joint probabilities determined from those trials on which the observer made an RS response. For each observer, the five parameters, $q, x, P_{A}, \beta_{A}$, and $\beta_{B}$, were determined, which minimized the sum of the squared deviations between the 18 values of $P\left(R_{S} \cap R_{j}\right)$, for $j=1$ or 2 , and the values predicted by the model. These parameter values are presented in Table 3, and in Figure 6, the predicted values of $P\left(R S \cap R_{j}\right)$ are shown. It is clear from Figure 6 that the predicted values are in close agreement with the data.

In Figure 5a, $\mathrm{P}(\mathrm{RS})$ as a function of $t$ is shown for the successiveness-only sessions, and in Figure $5 \mathrm{~b}$ for the joint sessions. The lines represent the predictions of the model using the appropriate parameters shown in Table 3. The successiveness function is clearly dependent upon the task requirement. Not surprisingly, the observer is more likely to make an $\mathrm{RS}$ response if he is also required to indicate order (joint sessions) than if he is not. What is of interest is that although the value of the "guessing" parameter, $\beta$. depends upon the task requirement, the value of the parameter representing the source of errors, q, does not. For two observers, an identical value of $q$ is obtained from the two tasks; for the remaining observer, the discrepancy is only $3 \mathrm{msec}$. Furthermore, the values of $q$ in Table 3 are well within the range reported previously by Allan and Kristofferson (1974) and Kristofferson (1967b).

While the values of $q$ in Table 3 are consistent with those reported previously, the slope of the successiveness discrimination function is considerably smaller. In the previous studies, only $S_{0}$ and $S_{1}$ patterns were presented, and in general the value of $\mathrm{P}_{\mathrm{A}}$ was close to 1.0. Examination of the equation for the successiveness function in Table 2 shows that the slope is a function of $\mathrm{P}_{\mathrm{A}}$. For $\mathrm{PA}_{\mathrm{A}}=1.0, \mathrm{P}\left(\mathrm{RS}_{\mathrm{S}} \mid \mathrm{S}_{1}\right)$ reaches 1.0 when $t=x+q$; for $P_{A}<1.0$, $P\left(R S \mid S_{1}\right)$ reaches 1.0 when $t=x+2 q$. Thus, within the context of the model, it is not surprising that the difference limen is larger in a successiveness discrimination task employing positive and negative values of $t$ than in a task using only positive values or only negative values.

Using the values of the five parameters estimated on the basis of $P\left(R S \cap R_{1}\right)$ and $P\left(R S \cap R_{2}\right)$, the value of $\lambda$ was determined which minimized the sum

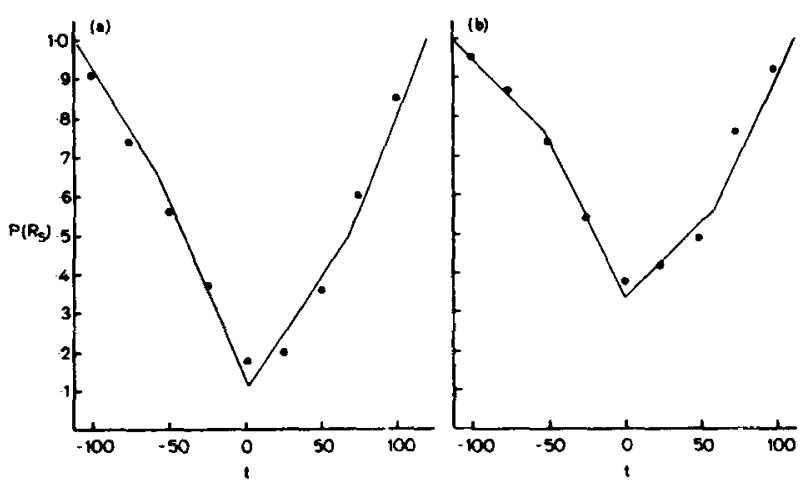

Figure 5. $P\left(R_{S}\right)$ as a function of $t$. The lines represent the predictions of the attention-switching model. The data in (a) were obtained during the successiveness-only sessions, the data in (b) during the joint sessions. 
Table 4

The Attention-Switching Model Joint Function

\begin{tabular}{|c|c|c|c|}
\hline $\mathrm{t}$ & $\mathbf{P}\left(\mathbf{R}_{\mathbf{S}} \cap \mathbf{R}_{1}\right)$ & $\mathbf{P}\left(\mathbf{R}_{\mathbf{S}} \cap \mathbf{R}_{2}\right)$ & $\mathbf{P}\left(\mathbf{R}_{\boldsymbol{O}} \cap \mathbf{R}_{1}\right)$ \\
\hline $\begin{array}{l}x+q \leqslant t \leqslant t+2 q \\
x \leqslant t \leqslant t+q \\
x-q \leqslant t \leqslant x \\
x-2 q \leqslant t \leqslant x-q\end{array}$ & $\begin{array}{l}\alpha\left(1-\mathbf{P}_{\mathbf{A}}\right)+\mathbf{P}_{\mathbf{A}} \\
\alpha \mathbf{P}_{\mathbf{A}}\left(1-\beta_{\mathbf{A}}\right)+\beta_{\mathbf{A}} \mathbf{P}_{\mathbf{A}} \\
\beta_{\mathbf{A}} \mathbf{P}_{\mathbf{A}}{ }_{-\alpha \beta_{\mathbf{A}}} \mathbf{P}_{\mathbf{A}}+\dot{\beta}_{\mathbf{A}} \mathbf{P}_{\mathbf{A}}\end{array}$ & $\begin{array}{l}-\alpha \beta_{B}\left(1-P_{A}\right)+\beta_{B}\left(1-P_{A}\right) \\
\beta_{B}\left(1-P_{A}\right) \\
\alpha\left(1-P_{A}\right)\left(1-\beta_{B}\right)+\beta_{B}\left(1-P_{A}\right) \\
\alpha P_{A}+\left(1-P_{A}\right)\end{array}$ & $\begin{array}{l}-\alpha \lambda\left(1-\beta_{\mathbf{B}}\right)\left(1-\mathbf{P}_{\mathbf{A}}\right)+\lambda\left(1-\beta_{\mathbf{B}}\right)\left(1-\mathbf{P}_{\mathbf{A}}\right) \\
-\alpha \lambda\left(1-\beta_{\mathbf{A}}\right) \mathbf{P}_{\mathbf{A}}+\lambda\left[1-\mathbf{P}_{\mathbf{A}}\left(\beta_{\mathbf{A}}-\beta_{\mathbf{B}}\right)-\beta_{\mathbf{B}}\right] \\
-\alpha \lambda\left(1-\beta_{\mathbf{B}}\right)\left(1-\mathbf{P}_{\mathbf{A}}\right)+\lambda\left[1-\mathbf{P}_{\mathbf{A}}\left(\beta_{\mathbf{A}}-\beta_{\mathbf{B}}\right)-\beta_{\mathbf{B}}\right] \\
-\alpha \lambda\left(1-\beta_{\mathbf{A}}\right) \mathbf{P}_{\mathbf{A}}+\lambda\left(1-\beta_{\mathbf{A}}\right) \mathbf{P}_{\mathbf{A}}\end{array}$ \\
\hline
\end{tabular}

of the squared discrepancies between $P\left(R_{0} \cap R_{1}\right)$ and the values predicted by the model (Table 4 ). The estimated values of $\lambda$ are presented in Table 3 for each observer, and the predicted function is shown in Figure 7.

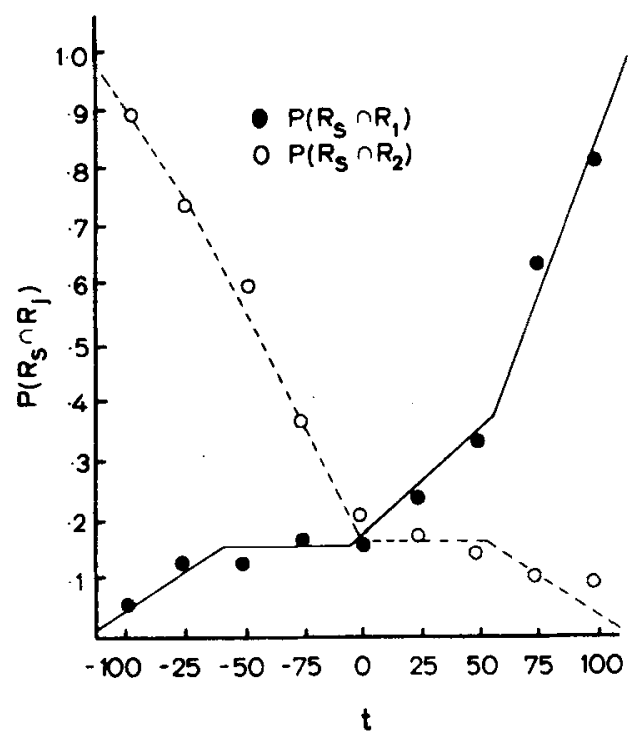

Figure 6. $P\left(R_{S} \cap R_{1}\right)$ and $P\left[R S \cap R_{2}\right)$ as a function of $t$. The lines represent the predictions of the attention-switching model.

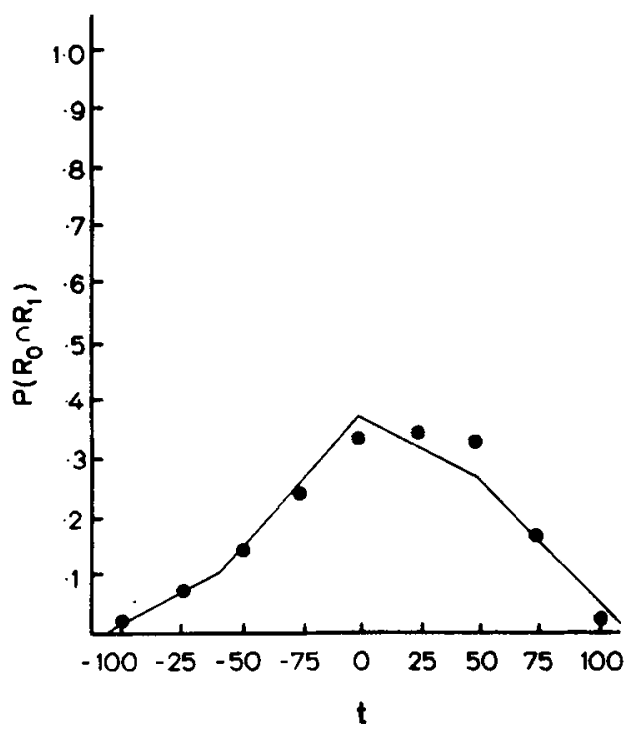

Figure 7. $P\left(R_{0} \cap R_{1}\right)$ as a function of $t$. The lines represent the predictions of the attention-switching model.
According to the model, the observer only makes an $R_{0}$ response on trials when he did not have any order information. Since the parameter $\lambda$ represents the observer's preference for making an $R_{1}$ response on such trials, the value of $\lambda$ should be constant and independent of $t$. The reasonable correspondence between the data and the model shown in Figure 7 , generated by a constant value of $\lambda$, can be taken to support the postulate that there is an internal state in which no order information is available. If the observer indicates that he cannot distinguish the two offsets from simultaneous offsets (an $R_{0}$ response), he is accurately admitting that he is incapable of transmitting order information.

The attention-switching model for the order-only sessions states that the observer will always make the correct order response when he perceives the two offsets as successive. When he does not perceive the two off'sets as successive, he will make an $R_{1}$ response with probability $\theta_{i}$, for $i$ equal to $A$ or $B$. The predicted relationship between $P\left(R_{1}\right)$ and $t$ is available in Table 5. For each observer, the value of $q$ estimated from the successiveness and joint sessions was used to obtain the predicted values for the order-only sessions (an average value of $53 \mathrm{msec}$ was used for T.M.). Since all observers indicated a small value of $x$ during the other session types, this parameter was fixed at $0 \mathrm{msec}$. The predicted order functions for $\theta_{A}=\theta_{B}=\theta$ are shown in Figure 8 . The values of $\mathrm{P}_{A}$ and $\theta$, and the proportion of the total variance in $P\left(R_{1}\right)$ accounted for by the model, are available in Table 3 . On the average, the two-parameter fits are quite good.

Again, given that the values of $P_{A}$ for the order-only sessions are less than 1.0, the relatively large difference limen for order judgments is expected within the context of the model.

\section{CONCLUDING COMMENTS}

The relationship between judgments of successiveness and judgments of order was examined. The approach taken was to examine the data in terms of models which assume that the perception of successiveness is necessary and sufficient for the perception of correct order. It was demonstrated that successiveness and order judgments cannot be jointly explained by perceptual latency models which assume that both judgments are based on the same internal event. This is the case regardless of the form of the perceptual latency distribution, and for either a 
Table 5

The Attention-Switching Model Order Function

\begin{tabular}{ll}
\hline \multicolumn{1}{c}{$t$} & \multicolumn{1}{c}{$P\left(R_{1}\right)$} \\
\hline$x+q \leqslant t \leqslant x+2 q$ & $\alpha\left(1-P_{A}\right)\left(1-\theta_{B}\right)+P_{A}+\theta_{B}\left(1-P_{A}\right)$ \\
$x \leqslant t \leqslant x+q$ & $\alpha P_{A}\left(1-\theta_{A}\right)+P_{A}\left(\theta_{A}-\theta_{B}\right)+\theta_{B}$ \\
$x-q \leqslant t \leqslant x$ & $-\alpha\left(1-P_{A}\right) \theta_{B}+P_{A}\left(\theta_{A}-\theta_{B}\right)+\theta_{B}$ \\
$x-2 q<t \leqslant x-q$ & $-\alpha P_{A} \theta_{A}+P_{A} \theta_{A}$ \\
\hline
\end{tabular}

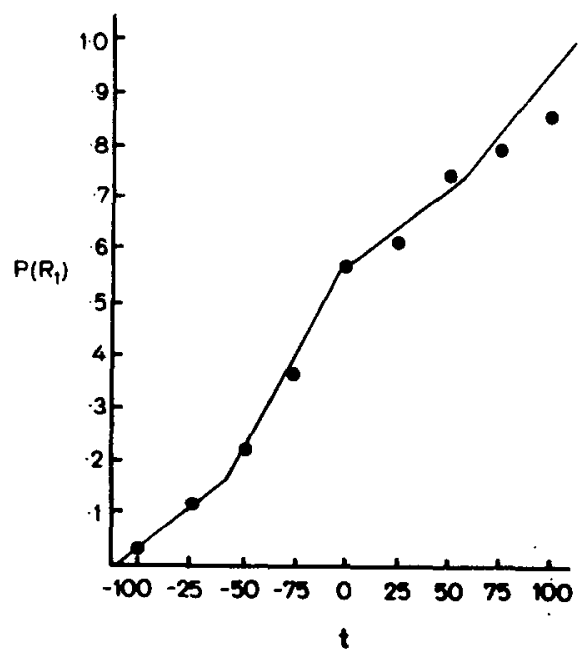

Figure 8. $P\left(R_{1}\right)$ as a function of $t$ for the order-only seasions. The lines represent the predictions of the attention-switching model.

threshold or a nonthreshold decision process. The attention-switching model was able to account for the data from the joint sessions. Thus, the predictions of one model, which holds that the perception of successiveness is sufficient for the perception of order, are consistent with the obtained data.

The most serious criticisms of the attentionswitching model have stemmed from the work of Baron $(1969,1971,1973)$. Allan (1975) noted that Baron $(1969,1971)$ incorrectly classified the attention-switching model as a discrete moment model, and demonstrated that, while Baron (1971) was correct that his second-guess data provide grounds for rejecting discrete moment models, the data are not inconsistent with the basic assumptions of the attention-switching model.
The attention-switching model was proposed as an explanation of errors in temporal judgments of stimuli in independent input channels. In all of the studies by Kristofferson and by Allan, the two stimulus events have been in different sensory modalities (a light and a tone). In all of Baron's studies, the stimuli have been unimodal. It is conceivable that his stimuli can be attended to simultaneously, and that the source of errors in such a situation is therefore not the waiting time to switch attention. The different hypotheses consistent with results from bimodal and unimodal stimuli deserve further investigation.

\section{REFERENCES}

Allan, L. G. Second guesses and the attention-switching model for successiveness discrimination. Perception \& Psychophysics, 1975, 17, 65-68.

Allan, L. G., \& Kristofferson, A. B. Successiveness discrimination: Two models. Perception \& Psychophysics, 1974. 15, 37.46.

BARON, J. Temporal ROC curves and the psychological moment. Psychonomic Science, 1969, 15, 299-300.

BARON, J. The threshold for successiveness. Perception \& Psychophysics, 1971, 10, 201-207.

BARON, J. Division of attention in successiveness discrimination. In S. Kornblum (Ed.), Attention and performance IV. New York: Academic Press, 1973. Pp. 703-711.

Gibbon, J., \& Rutschmans, R. Temporal order judgment and reaction time. Science, 1969, 165, 413-415.

Hirsh, I. J. Auditory perception of temporal order. Journal of the Acoustical Society of America, 1959, 31, 759-767.

Kristofferson, A. B. Attention and psychophysical time. Acta Psychologica, 1967, 27, 93-100. (a)

Kristofferson, A. B. Successiveness discrimination as a two-state quantal process. Science, 1967, 158, 1337-1339. (b)

KRistofferson, A. B. Attention. In R. M. Patton, T. A. Tanner. Jr., J. Markowitz, and J. A. Swets (Eds.), Applications of research on human decision-making. Washington, D.C: NASA, 1970. Pp. 49-64.

Kristofferson, A. B., \& Allan, L. B. Successiveness and duration discrimination. In S. Kornblum (Ed.), Attention and performance IV. New York: Academic Press, 1973. Pp. 737-749.

Sternberg, S., \& KNoll, R. L. The perception of temporal order: Fundamental issues and a general model. In S. Kornblum (Ed.), Attention and performance IV. New York: Academic Press, 1973. Pp. 629-685.

Wallach, H., Newman, E. B., \& Rosenzweig, M. R. The precedence effect in sound localization. American Journal of Psychology, 1949, 52, 315-336.

(Received for publication July 1974; revision accepted A pril 16, 1975.) 